\title{
Dietary fatty acid distribution modifies obesity risk linked to the rs9939609 polymorphism of the fat mass and obesity-associated gene in a Spanish case-control study of children
}

\author{
Adriana Moleres ${ }^{1}$, M. Carmen Ochoa ${ }^{2}$, Tara Rendo-Urteaga ${ }^{1}$, M. Angel Martínez-González ${ }^{3}$, \\ M. Cristina Azcona San Julián ${ }^{4}$, J. Alfredo Martínez ${ }^{1}$, Amelia Marti ${ }^{1 *}$ on behalf of GENOI† \\ ${ }^{1}$ Department of Nutrition, Food Science, Physiology and Toxicology, University of Navarra, C/Irunlarrea s/n, \\ Pamplona 31008, Spain \\ ${ }^{2}$ Center for Applied Medical Research, University of Navarra, Pamplona, Spain \\ ${ }^{3}$ Department of Preventive Medicine and Public Health, University of Navarra, Pamplona, Spain \\ ${ }^{4}$ Paediatric Endocrinology Unit, Department of Pediatrics, Clinica Universidad de Navarra, Pamplona, Spain
}

(Received 1 December 2010 - Revised 21 April 2011 - Accepted 23 May 2011 - First published online 29 July 2011)

\section{Abstract}

The rs9939609 polymorphism of the fat mass and obesity-associated (FTO) gene has been widely associated with childhood obesity in several European cohorts. This association appears to be dependent on dietary macronutrients. Therefore, the aim of the present study was to evaluate whether dietary fatty acid intake distribution could interact with this FTO genetic variation and obesity in a Spanish case-control study of children and adolescents. A total of 354 Spanish children and adolescents aged 6-18 years (49\% males) were genotyped for the rs9939609 variant of the FTO gene. Anthropometric parameters were taken and energy intake was measured. We observed an interaction between the consumption of SFA (percentage of total energy) and PUFA:SFA ratio and obesity risk linked to the rs9939609 SNP of the FTO gene. In the study population of the present study, the risk allele carriers consuming more than $12.6 \%$ SFA (of total energy) had an increased obesity risk compared with TT carriers. In a similar way, A allele carriers with an intake ratio lower than 0.43 PUFA:SFA presented a higher obesity risk than TT subjects. In summary, the present study reports for the first time the influence of dietary fatty acid distribution on the effect of the rs9939609 polymorphism of the FTO gene on children and adolescents' obesity risk.

Key words: Childhood obesity: Fat mass and obesity-associated gene: Fatty acid intake: Obesity risk

According to the estimates from the International Obesity Taskforce, at least 155 million school-aged children worldwide are overweight or obese. Within that figure, approximately $30-45$ million are classified as obese, accounting for $2-3 \%$ of the children aged 5-17 years in the world ${ }^{(1)}$. The European Association for the Study of Obesity estimates that 16-22\% of European adolescents aged between 14 and 17 years are overweight or obese, with an annual increase of the prevalence of approximately $2 \%$ in the 1990 s and early $2000 \mathrm{~s}^{(2)}$. Childhood obesity is usually accompanied by associated comorbidities such as type 2 diabetes mellitus, insulin resistance or the metabolic syndrome $e^{(3,4)}$.

In relation to the genetics of obesity, SNP of the fat mass and obesity-associated (FTO) gene have recently been strongly associated with excessive body weight for height and adiposity. Thus, FTO has been identified as a candidate gene contributing to childhood obesity in several European cohorts $^{(5-7)}$. This gene, located in human chromosome 16, has been proposed to have a nucleic acid demethylation activity that might regulate the expression of genes involved in metabolism, leading to obesity ${ }^{(8)}$.

Carriers of the minor frequency A allele of rs9939609, one of the most prevalent FTO genetic variants, have previously been identified with greater BMI in several studies ${ }^{(9-11)}$. Moreover, this polymorphism has been reported to interact with energy intake patterns in children, with carriers of the minor A allele consuming more fat and total energy than non-carriers ${ }^{(9,12,13)}$.

With regard to specific macronutrient dietary composition, Sonestedt et $a l^{(14)}$ have shown that fat and carbohydrate intake modifies the association between the rs9939609 genetic variation in the FTO gene and obesity in a Swedish population.

Abbreviations: FTO, fat mass and obesity associated; SDS, standard deviation score.

*Corresponding author: Dr A. Marti, fax +34 948425740, email amarti@unav.es

† Other members of GENOI are: M. Chueca, M. Oyarzabal, A. Patiño, R. Pelach and M. J. Moreno-Aliaga. 
Therefore, the aim of the present study was to study whether dietary fat composition modifies the association between obesity risk and the FTO genetic variant (rs9939609) in a Spanish case-control study of children and adolescents.

\section{Subjects and methods}

The study population included 354 Spanish children and adolescents (49\% males) aged 6-18 years who were enrolled in a case-control study (Grupo Navarro de Estudio de la Obesidad Infantil; GENOI). The subjects were recruited from the Paediatric Departments at the Virgen del Camino Hospital, Clínica Universidad de Navarra and other Primary Care Centres in Navarra (Spain). Cases were subjects with a BMI above the ninety-seventh percentile of the Spanish BMI reference data for age and $\operatorname{sex}^{(15)}$. Exclusion criteria were exposure to hormonal treatment or development of secondary obesity due to endocrinopathy or serious intercurrent illness. Controls were healthy subjects with a BMI below the ninety-seventh percentile of the same reference.

Written consent to participate was requested from both parents and adolescents aged above 12 years. The study protocol was performed in accordance with the ethical standards of the Declaration of Helsinki (as revised in Hong Kong in 1989, in Edinburgh in 2000 and in South Korea in 2008), and was approved by the ethics committee of the University of Navarra.

\section{Procedures}

Trained researchers conducted face-to-face interviews with participants and their parents based on standardised procedures. A semi-quantitative FFQ, previously validated in Spain $^{(16)}$, containing 132 food items was filled in, in order to evaluate dietary patterns. Complete data were available for 288 children and adolescents (53\% males). Familial medical history was collected using a specific questionnaire. Weight and height were measured with an electronic scale (type SECA 861; SECA, Birmingham, UK) and a telescopic heightmeasuring instrument (type SECA 225; SECA), respectively, to establish BMI-standard deviation score (SDS) according to the criteria of Cole et al. ${ }^{(17)}$. Skinfolds were measured with a Holtain skinfold calliper (Holtain, Crosswell, Crymych, Pembs, UK) and waist and hip circumferences with a flexible non-stretchable measuring tape (type SECA 200; SECA) using validated protocols. Percentage of body fat was determined by bioelectrical impedance (TBF-300A Body Composition Analyzer/Scale; TANITA, Tokyo, Japan). Venous blood samples were collected to obtain DNA samples.

\section{Genotyping}

DNA was extracted from the buffy coat fraction using a commercial kit (MasterPure ${ }^{\mathrm{TM}}$; Epicentre, Madison, WI, USA). All the subjects were genotyped for the rs9939609 polymorphism of the FTO gene using Taqman SNP allelic discrimination (ABI PRISM 7900; Applied Biosystems, Madrid, Spain). The probes and the primers for these assays were designed by Applied Biosystems. Replicate quality control samples were included in every genotyping plate with more than $99 \%$ concordance.

\section{Statistical analysis}

Statistical analyses were performed using the Statistical Package for the Social Sciences software 15.0 (SPSS Inc., Chicago, IL, USA). A $\chi^{2}$ test was used to evaluate the Hardy-Weinberg equilibrium. The Kolmogorov-Smirnov test was used to determine variable distribution.

The differences in anthropometric, biochemical and energy intake variables between the polymorphism genotypes were tested with ANCOVA adjusted for age and sex (for normally distributed variables) or the Mann-Whitney $U$ test. Multivariate logistic regression models were fitted to assess the association between the genotypes and obesity risk after adjusting for confounder factors. For the evaluation of the association between the rs9939609 SNP of the FTO gene and obesity risk, fat intake values were dichotomised by the median. The level of probability was set at $P<0.05$ as statistically significant.

\section{Result}

The distribution of genotypes for the rs9939609 polymorphism of the FTO gene variant was in Hardy-Weinberg equilibrium in the study population. The minor A allele frequency was $0 \cdot 46$. The prevalence of the polymorphism was higher in cases compared to controls, both in the codominant and in the dominant model, with a marginal statistical significance ( $P=0.060$ and 0.098 , respectively, $\chi^{2}$ test; Table 1 ).

As expected, anthropometric measurements such as weight, BMI-SDS, waist and hip circumferences or fat mass were significantly different $(P<0 \cdot 001)$ between cases $(n 208)$ and controls ( $n$ 146). With regard to energy intake, consumption of PUFA was significantly higher in the control group $(P=0.002)$, whereas obese subjects had a higher intake of MUFA $(P<0 \cdot 001$; Table 1$)$.

Logistic regression models were performed to study the association between this SNP of the FTO gene and obesity risk, including a recessive (T carriers $v$. AA), a dominant (TT $v$. A carriers) and a codominant (TT $v$. TA $v$. AA) model adjusted for age, sex, total energy $(\mathrm{kJ})$ and total fat $(\mathrm{g})$ intake. The OR for obesity increased for each additional A allele in the genotype (Table 2). Children and adolescents homozygous for the mutation (AA) had higher risk of becoming obese than those without the mutation (OR 1.89; $95 \%$ CI 1.06, 3.30).

Next, we analysed the effect of the rs9939609 mutation of the FTO gene on obesity risk, depending on the fatty acid consumption. A relationship between SFA intake and obesity was found (Fig. 1(a)). After dichotomising for the median, we observed that a higher SFA intake $(\geq 12.6 \%)$ contributes to a greater obesity risk. A statistically significant interaction term (FTO SNP $\times$ SFA consumption) on BMI-SDS was also identified $(P$ for interaction $=0 \cdot 035)$. In subjects with a lower intake of SFA $(<12.6 \%$ of total energy), the mutation did not seem to affect BMI-SDS, whereas A allele carriers with a 
Table 1. Baseline characteristics and prevalence of the rs9939609 polymorphism of the fat mass and obesity-associated gene in children and adolescent obese and control subjects

(Mean values with their standard error of mean, after adjusting for age and sex and number of subjects and percentage values)

\begin{tabular}{|c|c|c|c|c|c|}
\hline & \multicolumn{2}{|c|}{ Obese ( $n$ 208) } & \multicolumn{2}{|c|}{ Control (n 146) } & \multirow[b]{2}{*}{$P$} \\
\hline & Mean & SEM & Mean & SEM & \\
\hline Males (\%) & \multicolumn{2}{|c|}{50} & \multicolumn{2}{|c|}{49} & \\
\hline Age (years) & $11 \cdot 6$ & 0.20 & 11.5 & 0.17 & 0.608 \\
\hline Weight $(\mathrm{kg})$ & 63.7 & 0.70 & $43 \cdot 1$ & 0.77 & $<0.001$ \\
\hline $\mathrm{BMI}\left(\mathrm{kg} / \mathrm{m}^{2}\right)$ & $27 \cdot 4$ & 0.20 & $19 \cdot 0$ & 0.22 & $<0.001$ \\
\hline BMI-SDS & 3.53 & 0.46 & 0.28 & 0.42 & 0.001 \\
\hline Waist circumference $(\mathrm{cm})$ & $86 \cdot 4$ & 0.77 & 65.9 & 0.67 & $<0.001$ \\
\hline Hip circumference $(\mathrm{cm})$ & 99.5 & 0.70 & $81 \cdot 7$ & 0.62 & $<0.001$ \\
\hline Waist:hip ratio & 0.87 & 0.005 & 0.81 & 0.004 & $<0.001$ \\
\hline Fat mass (\%) & 34.5 & 0.47 & $18 \cdot 4$ & 0.53 & $<0.001$ \\
\hline Tricipital skinfold $(\mathrm{mm})$ & $25 \cdot 4$ & 0.37 & $15 \cdot 7$ & 0.41 & $<0.001$ \\
\hline \multicolumn{6}{|l|}{ Dietary fat consumption } \\
\hline$n$ & \multicolumn{2}{|c|}{155} & \multicolumn{2}{|c|}{133} & \\
\hline Total fat (\% TE) & $37 \cdot 7$ & 0.40 & $36 \cdot 6$ & 0.40 & 0.057 \\
\hline PUFA (\% TE) & 5.50 & 0.12 & 6.03 & 0.13 & 0.004 \\
\hline SFA (\% TE) & $12 \cdot 9$ & 0.21 & $13 \cdot 1$ & 0.22 & 0.351 \\
\hline MUFA (\% TE) & $16 \cdot 0$ & 0.22 & $14 \cdot 8$ & 0.24 & $<0.001$ \\
\hline PUFA:SFA & 0.44 & 0.01 & 0.47 & 0.01 & 0.079 \\
\hline$n-3(\% \mathrm{TE})$ & 0.47 & 0.03 & 0.48 & 0.03 & 0.824 \\
\hline Trans-fatty acids (\% TE) & 0.98 & 0.07 & 0.98 & 0.07 & 0.986 \\
\hline \multicolumn{6}{|l|}{ rs9939609 } \\
\hline \multicolumn{6}{|l|}{ TT } \\
\hline$n$ & \multirow{2}{*}{\multicolumn{2}{|c|}{$\begin{array}{c}53 \\
25.5\end{array}$}} & \multicolumn{2}{|c|}{49} & \\
\hline$\%$ & & & & & \\
\hline \multicolumn{6}{|l|}{ TA } \\
\hline$n$ & \multicolumn{2}{|c|}{$\begin{array}{c}106 \\
51.0\end{array}$} & \multicolumn{2}{|c|}{76} & \\
\hline$\%$ & & & & & 0.060 \\
\hline \multicolumn{6}{|l|}{ AA } \\
\hline$n$ & \multicolumn{2}{|c|}{49} & \multicolumn{2}{|c|}{21} & \\
\hline$\%$ & \multicolumn{2}{|c|}{$23 \cdot 6$} & \multicolumn{2}{|c|}{14.4} & \\
\hline
\end{tabular}

SDS, standard deviation score; TE, total energy.

higher intake of SFA had a significantly higher BMI-SDS $(P=0.009)$ than TT subjects (Fig. 2).

Furthermore, as seen in Fig. 1(b), having a PUFA:SFA intake ratio higher than 0.43 (median) seems to protect against obesity risk. We observed an interaction between the rs9939609 SNP of the FTO gene (dominant model) and PUFA:SFA intake ratio ( $P$ for interaction $=0.034$ ), and a borderline statistical interaction between the rs9939609 SNP of FTO and SFA consumption ( $P$ for interaction $=0 \cdot 080$ ).

Table 2. Obesity associated with the rs9939609 polymorphism of the fat mass and obesity-associated gene in codominant, dominant and recessive models

(Odds ratios and $95 \%$ confidence intervals adjusted for age and sex)

\begin{tabular}{lllc}
\hline rs9939609 & OR & $95 \% \mathrm{Cl}$ & $P$ \\
\hline $\begin{array}{l}\text { Codominant } \\
\text { TT }\end{array}$ & 1 & & 0.059 \\
TA & 1.28 & $0.78,2.09$ & 0.321 \\
AA & 2.20 & $1.15,4.21$ & 0.018 \\
Dominant & 1 & & \\
TT & 1.47 & $0.92,2.03$ & 0.106 \\
TA+AA & 1 & & \\
Recessive & 1.89 & $1.06,3.30$ & 0.031 \\
TT + TA & & & \\
AA & & & \\
\hline
\end{tabular}

\section{Discussion}

In the present study, we confirm the previously reported association between the rs9939609 polymorphism of the FTO gene with obesity in a case-control population of children and adolescents. Moreover, we characterise the role of dietary fat intake distribution and its interaction with this genetic variant as a risk factor for obesity.

The frequency of the minor A allele was 0.46 , similar to those described in other Spanish populations ${ }^{(18)}$ and other European cohorts of children and adolescents ${ }^{(19,20)}$.

Case-control studies have been demonstrated to be a suitable tool to screen the effects that a genetic polymorphism may have on obesity development. It has been previously reported that the accuracy of the phenotype measurement is crucial in the ability to detect gene-environment interactions for traits such as $\mathrm{BMI}^{(21)}$. On this point, the subjects of the present study were a homogeneous sample of cases and controls of similar age and belonging to the same social environment. As expected, control subjects showed statistically significant differences compared with obese children and adolescents in all measured anthropometric traits.

The rs9939609 polymorphism of the FTO gene has been largely related to greater adiposity. With regard to this observation, the data from the present study showed a significant 

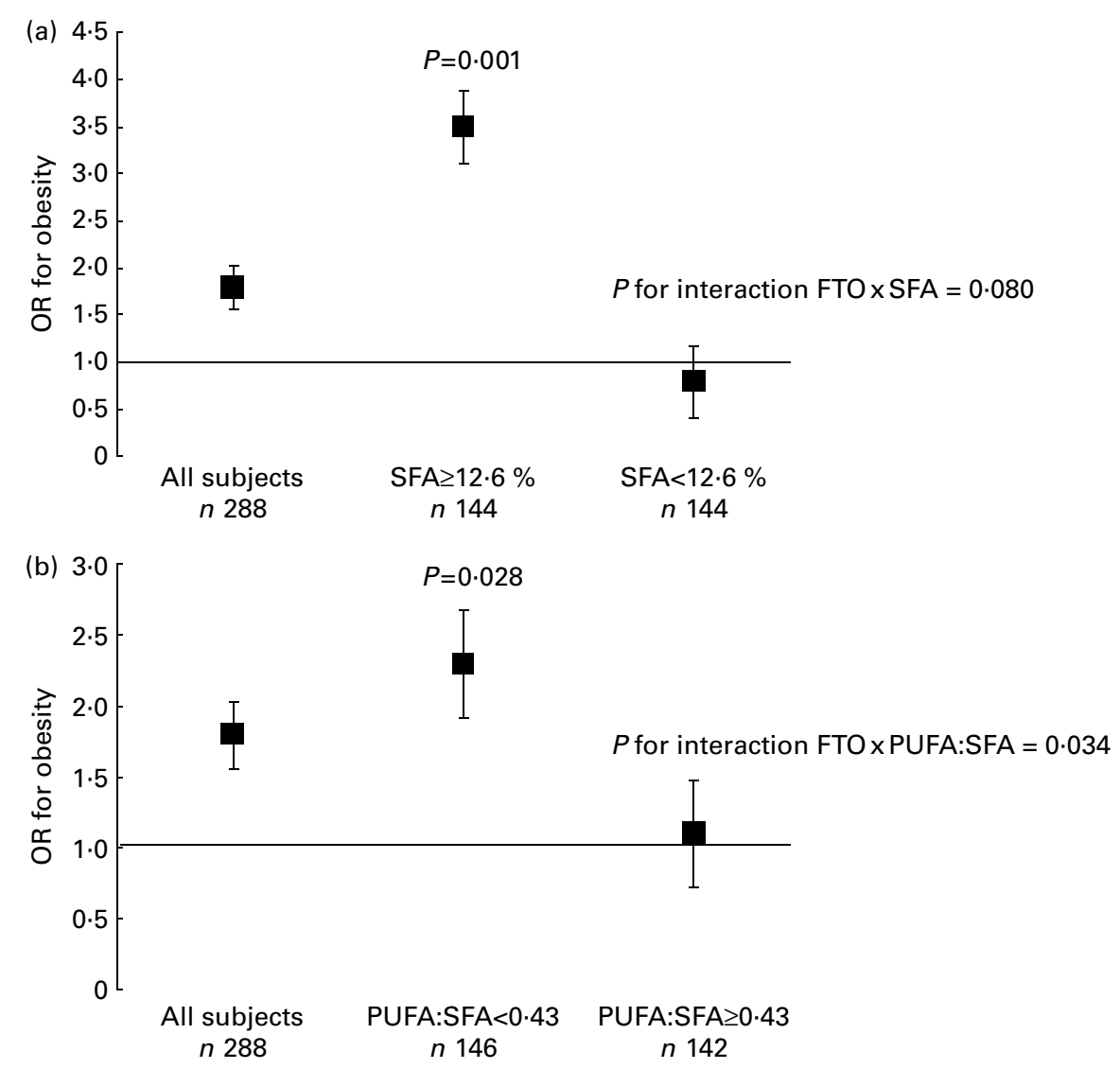

Fig. 1. OR for obesity risk in children and adolescents depending on the consumption of (a) SFA (percentage of total energy, dichotomised by the median) and (b) PUFA:SFA ratio (dichotomised by the median) and the rs9939609 SNP of the fat mass and obesity associated (FTO) gene (dominant model). OR are adjusted for age and sex. TT subjects are considered as the reference group $(\mathrm{OR}=1)$.

increase in obesity risk per A allele present in the genotype. Homozygous carriers for the mutation are at a greater risk of developing obesity than non-carriers (OR 1.89; 95\% CI 1.06, 3.30). This increase in obesity risk was similar to that observed by Wardle et $a l^{(22)}$ in a population of British children and slightly higher than those observed by other authors ${ }^{(10,23,24)}$, both in children and adult cohorts.
The present results confirm that the effect of the mutation is influenced by dietary fatty acid composition. The percentage PUFA, SFA and PUFA:SFA ratio over total energy intake modulates obesity risk associated with the rs9939609 polymorphism of the FTO gene. Specifically, children and adolescents carrying the A allele of the mutation and consuming more than $12.6 \%$ SFA (as percentage of total energy) had an increased

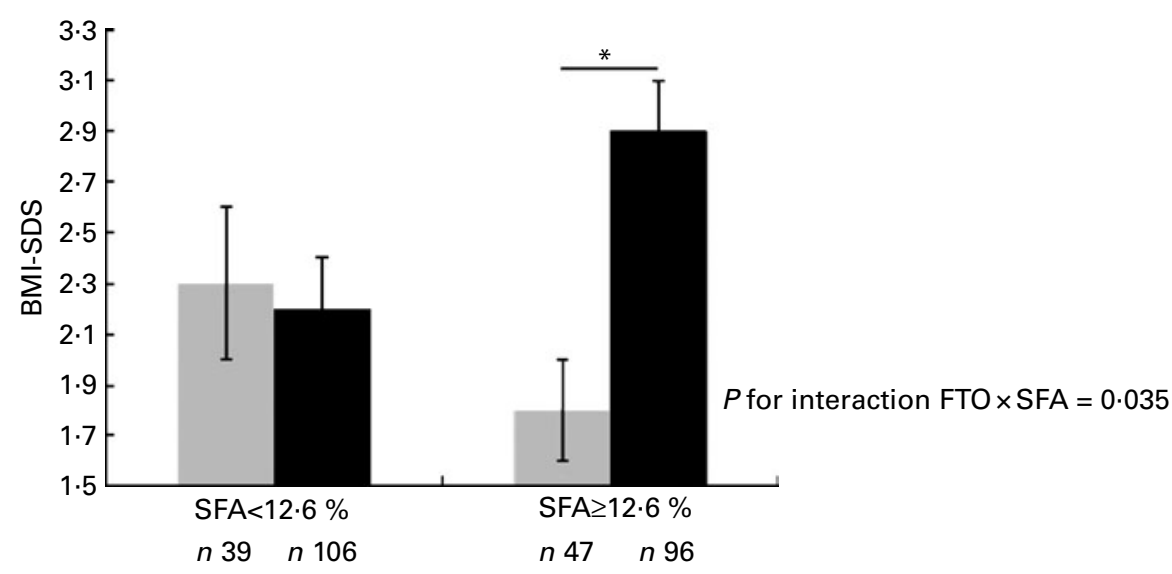

Fig. 2. BMI-standard deviation score (SDS) of children and adolescents according to SFA consumption (percentage of total energy, dichotomised by the median) and the presence of the fat mass and obesity associated (FTO) rs9939609 polymorphism in a dominant model. Values are means, with their standard errors represented by vertical bars. $\square$, TT; $\mathbf{\square}$, A carriers. * Mean values were significantly different $(P<0.05)$. 
risk of being obese. In a similar way, we observed an interaction between the FTO polymorphism and the PUFA:SFA ratio. Indeed, subjects with the A allele of the FTO gene, with a PUFA:SFA ratio lower than 0.43 , had a 2.3 times higher risk of becoming obese than the non-carriers of the risk allele consuming a higher PUFA:SFA ratio.

Genetic associations and fatty acid-gene interactions are widely explored in obesity development and onset ${ }^{(25)}$. In this sense, Luan et al. showed an interaction between PUFA:SFA dietary intake ratio and the Pro12Ala polymorphism of the PPARG gene. In this study population, Ala carriers showed a decrease in their BMI as the PUFA:SFA ratio increased, whereas wild-type homozygotes were not affected by fatty acid intake ${ }^{(26)}$. Other gene variants have also showed effects of interactions with dietary fatty acid consumption on obesity risk. In 2007, Corella et al. ${ }^{(27)}$ found that a $A P O A 5$ gene variation modulates the effects of dietary fat intake on BMI and obesity risk in an adult American cohort. With regard to SFA, a recent study carried out in Mediterranean and Asian populations has also shown the effect of a genesaturated fat intake interaction on the association between the APOA2 promoter polymorphism and body weight ${ }^{(28)}$. All these studies demonstrate that fatty acid intake could affect obesity risk in a different way according to the individual genotype of the subject, and therefore, the study of these nutrient-gene interactions would be an important tool in obesity prevention and personalised nutrition.

With regard to the rs9939609 polymorphism of the FTO gene, there are some studies in children that link the SNP with a higher total energy and fat intake ${ }^{(9,12,13,20)}$ and diminished satiety sensation ${ }^{(22)}$. A recent study has showed that fat and carbohydrate intake modifies the association between the rs9939609 genetic variation of the FTO and obesity ${ }^{(14)}$, but specific dietary fatty acid composition and its interaction with this SNP and obesity risk has not been studied so far. A study carried out in our group by Razquin et $a l^{(18)}$ in high cardiovascular risk Spanish subjects aged 55-80 years showed that subjects carrying the A allele of the mutation gained significantly less weight compared to wild-type subjects (TT) after 3 years of intervention with the Mediterranean diet. This diet is characterised by a high intake of PUFA and a low intake of SFA, and confirms the implication of dietary fatty acid distribution in the relationship between FTO gene variant and body weight.

To our knowledge, this is the first study linking fatty acid intake with the FTO rs9939609 polymorphism on obesity risk. One limitation of the present study is sample size, and therefore, studies in larger populations, leading to a better understanding of how dietary macronutrients and the rs9939609 SNP of the FTO gene could interact and modify obesity risk, are needed to support the findings of the present study.

In summary, the present study confirms that the rs9939609 variation of the FTO gene is associated with a higher obesity risk in this case-control study of children and adolescents. Moreover, we report for the first time the influence of dietary fatty acid distribution on the effect of this polymorphism on obesity risk.

\section{Acknowledgements}

Research relating to the present study was funded by grants from the Navarra Government (Health and Education Department) and Línea Especial, Nutrición y Obesidad (University of Navarra). The scholarships to A. M. from the Navarra Government and to T. R.-U. from the University of Navarra's Asociación de Amigos are fully acknowledged. A. M., J. A. M. and M. A. M.-G. were involved in the design, funding and writing of the manuscript. M. C. A.-S. was involved in the design, conduct and writing of the manuscript. A. M., T. R.-U. and M. C. O. were involved in the conduct, analysis and writing of the manuscript. The authors have no competing interests.

\section{References}

1. IOTF (2005) Global picture of childhood obesity. http:// www.iotf.org/childhoodobesity.asp (accessed December 2010).

2. Baker JL, Farpour-Lambert NJ, Nowicka P, et al. (2010) Evaluation of the overweight/obese child - practical tips for the primary health care provider: recommendations from the Childhood Obesity Task Force of the European Association for the Study of Obesity. Obes Facts 3, 131-137.

3. Cali AM \& Caprio S (2008) Obesity in children and adolescents. J Clin Endocrinol Metab 93, S31-S36.

4. Ochoa MC, Santos JL, Azcona C, et al. (2007) Association between obesity and insulin resistance with UCP2-UCP3 gene variants in Spanish children and adolescents. Mol Genet Metab 92, 351-358.

5. Dina C, Meyre D, Gallina S, et al. (2007) Variation in FTO contributes to childhood obesity and severe adult obesity. Nat Genet 39, 724-726.

6. Frayling TM, Timpson NJ, Weedon MN, et al. (2007) A common variant in the FTO gene is associated with body mass index and predisposes to childhood and adult obesity. Science 316, 889-894

7. Haworth CM, Carnell S, Meaburn EL, et al. (2008) Increasing heritability of BMI and stronger associations with the FTO gene over childhood. Obesity (Silver Spring) 16, 2663-2668.

8. Fawcett KA \& Barroso I (2010) The genetics of obesity: FTO leads the way. Trends Genet 26, 266-274.

9. Cecil JE, Tavendale R, Watt P, et al. (2008) An obesityassociated FTO gene variant and increased energy intake in children. $N$ Engl J Med 359, 2558-2566.

10. Jacobsson JA, Danielsson P, Svensson V, et al. (2008) Major gender difference in association of FTO gene variant among severely obese children with obesity and obesity related phenotypes. Biochem Biophys Res Commun $\mathbf{3 6 8}$, 476-482.

11. Rendo T, Moleres A \& Marti Del Moral A (2009) Effects of the FTO gene on lifestyle intervention studies in children. Obes Facts 2, 393-399.

12. Timpson NJ, Emmett PM, Frayling TM, et al. (2008) The fat mass- and obesity-associated locus and dietary intake in children. Am J Clin Nutr 88, 971-978.

13. Wardle J, Llewellyn C, Sanderson S, et al. (2009) The FTO gene and measured food intake in children. Int $J$ Obes (Lond) 33, 42-45.

14. Sonestedt E, Roos C, Gullberg B, et al. (2009) Fat and carbohydrate intake modify the association between genetic variation in the FTO genotype and obesity. Am J Clin Nutr 90, 1418-1425. 
15. Sobradillo B (2004) Curvas y tablas de crecimiento (estudios longitudinal y transversal) (Growth Curves and Tables (Longitudinal and Transverse Studies)). Bilbao: Fundacion Faustino Orbegozo Eizaguirre.

16. Martin-Moreno JM, Boyle P, Gorgojo L, et al. (1993) Development and validation of a food frequency questionnaire in Spain. Int J Epidemiol 22, 512-519.

17. Cole TJ, Bellizzi MC, Flegal KM, et al. (2000) Establishing a standard definition for child overweight and obesity worldwide: international survey. BMJ 320, 1240-1243.

18. Razquin C, Martinez JA, Martinez-Gonzalez MA, et al. (2010) A 3-year intervention with a Mediterranean diet modified the association between the rs9939609 gene variant in FTO and body weight changes. Int J Obes (Lond) 34, 266-272.

19. Muller TD, Hinney A, Scherag A, et al. (2008) 'Fat mass and obesity associated' gene (FTO): no significant association of variant rs9939609 with weight loss in a lifestyle intervention and lipid metabolism markers in German obese children and adolescents. BMC Med Genet 9, 85 .

20. Tanofsky-Kraff M, Han JC, Anandalingam K, et al. (2009) The FTO gene rs9939609 obesity-risk allele and loss of control over eating. Am J Clin Nutr 90, 1483-1488.

21. Andreasen $\mathrm{CH}$, Stender-Petersen KL, Mogensen MS, et al. (2008) Low physical activity accentuates the effect of the
FTO rs9939609 polymorphism on body fat accumulation. Diabetes 57, 95-101.

22. Wardle J, Carnell S, Haworth CM, et al. (2008) Obesity associated genetic variation in FTO is associated with diminished satiety. J Clin Endocrinol Metab 93, 3640-3643.

23. Loos RJ \& Bouchard C (2008) FTO: the first gene contributing to common forms of human obesity. Obes Rev $\mathbf{9}$, 246-250.

24. Price RA, Li WD \& Zhao H (2008) FTO gene SNPs associated with extreme obesity in cases, controls and extremely discordant sister pairs. BMC Med Genet 9, 4.

25. Smith CE \& Ordovas JM (2010) Fatty acid interactions with genetic polymorphisms for cardiovascular disease. Curr Opin Clin Nutr Metab Care 13, 139-144.

26. Luan J, Browne PO, Harding AH, et al. (2001) Evidence for gene-nutrient interaction at the PPARgamma locus. Diabetes 50, 686-689.

27. Corella D, Lai CQ, Demissie S, et al. (2007) APOA5 gene variation modulates the effects of dietary fat intake on body mass index and obesity risk in the Framingham Heart Study. J Mol Med 85, 119-128.

28. Corella D, Tai ES, Sorli JV, et al. (2010) Association between the APOA2 promoter polymorphism and body weight in Mediterranean and Asian populations: replication of a gene-saturated fat interaction. Int J Obes (Lond) $\mathbf{3 5}$, 666-675.

>

